\title{
EBERHARD-TYPE THEOREMS WITH TWO KINDS OF POLYGONS
}

\author{
SEBASTIAN MANECKE
}

\begin{abstract}
Eberhard-type theorems are statements about the realizability of a polytope (or more general polyhedral maps) given the valency of its vertices and sizes of its polygonal faces up to a linear degree of freedom. We present new theorems of Eberhard-type where we allow adding two kinds of polygons and one type of vertices. We also hint towards a full classification of these types of results.
\end{abstract}

\section{Overview}

The classical Eberhard theorems are two results on the constructability of $r$ valent 3-polytopes for a given sequence $\left(p_{k}\right)_{k \geq 3}$, where $p_{k} \in \mathbb{N}=\{0,1,2, \ldots\}$ describes the number of occurrences of each $k$-gon. For us, a sequence $a$ will always be a function $\mathbb{N} \backslash\{0,1,2\} \rightarrow \mathbb{N}$ with finite support.

The original formulations were (see [4]):

Theorem 1.1 (Eberhard's theorem for 3 -valent 3 -polytopes). Let $\left(p_{3}, p_{4}, p_{5}\right.$, $\left.p_{7}, \ldots, p_{m}\right)$ be a sequence of natural numbers for which

$$
\sum_{\substack{3 \leq k \leq m \\ k \neq 6}}(6-k) \cdot p_{k}=12,
$$

holds. Then there exists a number $p_{6}$ and a 3-valent 3-polytope which has exactly $p_{k} k$-gons for each $3 \leq k \leq m$.

Theorem 1.2 (Eberhard's theorem for 4 -valent 3-polytopes). Let $\left(p_{3}, p_{5}, \ldots, p_{m}\right)$ be a sequence of natural numbers for which

$$
\sum_{\substack{3 \leq k \leq m \\ k \neq 4}}(4-k) \cdot p_{k}=8
$$

holds. Then there exists a number $p_{4}$ and a 4-valent 3-polytope which has exactly $p_{k} k$-gons for each $3 \leq k \leq m$.

If $p_{k}$ is the number of $k$-gons of a 3 -valent (resp. 4-valent) polytope, then (1D) (resp. (2) ) holds as an immediate consequence of Euler's relation and double counting of the number of edges. Thus these theorems answer the question under which condition a sequence $p$ that suffices the natural combinatorial conditions has a realization as a polytope.

In the 1970's Barnette, Ernest, Grünbaum, Jendrol', Jucovič, Trenkler and Zaks [7, 1, 3, 9, 6] extended these results to polyhedral maps, which are graph

Key words and phrases. Eberhard-type theorems; polyhedral maps; 3-polytopes; topological graph theory. 
embeddings on a closed topological 2-manifold (or surface) generalizing the combinatorics of 3-polytopes. In the two setups described by the classical Eberhard theorems there is now a complete characterization for which sequences $\left(p_{k}\right)_{k \geq 3}$ and $\left(v_{k}\right)_{k \geq 3}$ and surfaces $S$ there exists a polyhedral map on $S$ with $p_{k} k$-gons and $v_{k}$ $k$-valent vertices when choosing the value of $p_{6}$ and $v_{3}$, resp. $p_{4}$ and $v_{4}$, appropriately.

We want to call the sequences $p=\left(p_{k}\right)_{k \geq 3}$ and $v=\left(v_{k}\right)_{k \geq 3}$ which count the number of $k$-gons and $k$-valent vertices of a polyhedral map $M$ the $p$-vector and the $v$-vector of $M$ and call the pair $(p, v)$ to be realizable on a surface $S$, if there exists a polyhedral map $M$ on $S$ with $p$-vector $p$ and $v$-vector $v$.

An easy construction shows that we can in fact find infinitely many $p_{6}$ and $v_{3}$, resp. $p_{4}$ and $v_{4}$, such that $(p, v)$ is realizable as a polyhedral map on a fixed $S$. By using Euler's relation and an easy double counting argument one cannot increase $p_{6}$ and $v_{3}$, resp. $p_{4}, v_{4}$ independently from each other and thus one can deduce that there is a linear relation between these numbers. We want to propose the generalized Eberhard problem:

Question 1.3. Let $p=\left(p_{k}\right)_{k>3}, v=\left(v_{k}\right)_{k>3}, q=\left(q_{k}\right)_{k>3}$ and $w=\left(w_{k}\right)_{k>3}$ be sequences and $S$ be a surface. Does there exist infinitely many $c, d \in \mathbb{N}$ and $a$ polyhedral map on $S$ with $p$-vector $p+c \cdot q$ and v-vector $v+d \cdot w$ ?

Theorem 1.1 and its generalization to polyhedral maps answer this question for $q=(0,0,0,1,0, \ldots)$ and $w=(1,0, \ldots)$, whereas Theorem 1.2 and its generalizations answer this question for $q=(0,1,0 \ldots), w=(0,1,0, \ldots)$. It is easy to check, that the only missing possibility for $q$ and $w$ with exactly one non-zero entry, where such a statement can be true, is $q=(1,0, \ldots), w=(0,0,0,1,0, \ldots)$. This case is in fact just the dual of Theorem 1.1] and therefore all cases with exactly one non-zero entry in both $q$ and $w$ have been classified.

Question 1.3 for $q$, $w$ with more than one non-zero entry was first considered by DeVos et al. 2, who gave an answer in the case of $v=(1,0, \ldots), q=$ $(0,0,1,0,1,0, \ldots)$ and $w=(1,0, \ldots)$ for any surface. We will also consider similar theorems of this type in this article. To state them more easily, let us introduce the following notation: Define $[i]$ to be the sequence $a$ with $a_{i}=1$ and $a_{j}=0$ for $i \neq j$. We then set $\left[a_{k_{1}} \times k_{1}, a_{k_{2}} \times k_{2}, \ldots, a_{k_{n}} \times k_{n}\right]:=\sum_{i=1}^{n} a_{k_{i}}\left[k_{i}\right]$, where only entries $a_{k_{i}}$ not equal to zero occur. If $a_{k_{i}}=1$, we will just write $k_{i}$ instead of $1 \times k_{i}$.

In his master thesis [8, the author gave a complete answer to Question [1.3] in the case that $q$ has precisely two non-zero and coprime entries and $w$ has one nonzero entry. We will state the full theorem in Sec. 2 and give the ideas for the constructions used in the proofs in Sec. 3. The last Section, Sec. 4, will show how these constructions yield one case of the full statement in [8], that is to say, the following two theorems:

Theorem 1.4. Let $p$ and $v$ be a pair of admissible sequences for an orientable closed 2-manifold $S$ and $k \in \mathbb{N}$. Then there exists infinitely many $c, d \in \mathbb{N}$ for which there exists a polyhedral map on $S$ with p-vector $p+c \cdot[(3 k+1) \times 3,3 k+5]$ and $v$-vector $v+d \cdot[4]$.

Theorem 1.5. Let $p$ and $v$ be a pair of admissible sequences for an orientable closed 2-manifold $S$ and $k \in \mathbb{N}$. Then there exists infinitely many $c, d \in \mathbb{N}$ for which there exist a polyhedral map on $S$ with p-vector $p+c \cdot[(3 k+3) \times 3,3 k+7]$ and v-vector $v+d \cdot[4]$. 


\section{Polyhedral maps AND Generalized EBerhard PROBlems}

We will review basic notions from (topological) graph theory. A simple graph $G$ is a finite undirected graph without loops and multi-edges. If $G^{\prime}$ is a subgraph of $G$ this is denoted by $G^{\prime} \subseteq G$. We want to write $u_{1}-\cdots-u_{k}$ for paths and $u_{1}-\cdots-u_{k}-u_{1}$ for cycles. The valence of a vertex is the number of incident edges.

All of our graphs are considered to be embedded into a closed (topological) 2manifold, which we call surfaces for brevity. We assume our 2-manifolds to be oriented in this article. An embedding of a simple graph with vertices $V$, edges $E$ and faces $F$ is called a map, provided that $G$ is simple, every vertex $v \in V$ has valence at least 3 and every $f \in F$ is a closed 2-cell (i.e. homeomorphic to a disk). The faces of a map incident to $k$ edges (or equivalently, $k$ vertices) will be called $k$-gonal faces or simply $k$-gons. A map on a closed 2-manifold is called polyhedral, if for every two faces $f, f^{\prime}, f \neq f^{\prime}$ there is either no vertex, a single vertex or a single edge incident to both $f$ and $f^{\prime}$. In these cases the two faces are said to meet properly.

In Section 3 we will weaken the definition of a map to some extent to allow for 2 -valent vertices. This does not warrant a whole new definition, so we state it here for completeness and to avoid confusion.

An important property of polyhedral maps is that each edge contains exactly two vertices and is contained in exactly two faces. From this fact one can see that the concept of polyhedral maps dualizes perfectly, i.e. if an embedding is a polyhedral map, then the dual of the embedding is again a polyhedral map.

Example 2.1. We can view every 3-polytope as a map on a surface, where the graph of the map is the graph of the 3-polytope and the embedding is held by radial projection onto $\mathbb{S}^{2}$. In this context each face of the 3-polytope corresponds to one of the map. It is easy to see that this map is polyhedral, which gives rise to the property's name. Also note, that the dual map corresponding to a 3-polytope is the corresponding map of the dual polytope.

To further strengthen the link between 3-polytopes and polyhedral maps on the sphere $\mathbb{S}^{2}$, we mention the following two results:

Proposition 2.2. Every graph $G$ of a polyhedral map $M$ is 3-connected, i.e. $G$ has at least 3 vertices and the deletion of any 2 vertices leaves the graph connected.

Theorem 2.3 (Steinitz's theorem). A graph is the edge graph of a 3-polytope if and only if it is planar and 3-connected.

These two theorems combined yield that every polyhedral map on the sphere $\mathbb{S}^{2}$ comes from a 3-polytope and vice versa.

We will now turn our focus to Eberhard theorems for polyhedral maps on surfaces. The $p$-vector of a map $M$ on a 2 -manifold is the sequence $\left(p_{3}, \ldots, p_{m}\right)$, where $p_{k}$ denotes the number of faces with exactly $k$ vertices. Similarly the $v$-vector of $M$ is the sequence $\left(v_{3}, \ldots, v_{n}\right)$ where each $v_{k}$ is the number of vertices of $M$ with valence $k$.

A pair of sequences $(p, v)$ is said to be realizable as a polyhedral map on the closed 2-manifold $S$ (or short: realizable on $S$ ), if there exists such a map having $p$ as its $p$-vector and $v$ as its $v$-vector. In this language we can state the following two generalizations of Theorems 1.1 and 1.2, which will be central in our constructions: 
Theorem 2.4 (Jendrol', Jucovič [6], 1977). Each pair of sequences $p=\left(p_{3}, \ldots, p_{m}\right)$ and $v=\left(v_{3}, \ldots, v_{n}\right)$ is realizable on a closed orientable 2 -manifold with Euler characteristic $\chi$ for some $p_{6} \in \mathbb{N}, v_{3} \in \mathbb{N}$ if and only if

$$
\begin{aligned}
\sum_{k=3}^{m}(6-k) p_{k}+2 \sum_{k=4}^{n}(3-k) v_{k}=6 \chi, & \\
\sum_{\substack{k=3 \\
2 \nmid k}}^{m} p_{k} \neq 0 \quad \text { or } \quad \sum_{\substack{k=4 \\
3 \nmid k}}^{n} v_{k} \neq 1 & \text { if } \chi=2, \\
p \neq[5,7] \quad \text { or } \quad v \neq\left[v_{3} \times 3\right] & \text { if } \chi=0 .
\end{aligned}
$$

Theorem 2.5 (Barnette, Grünbaum, Jendrol', Jucovič, Zaks [7, 1, 3, 9], 1973). Each pair of sequences $p=\left(p_{3}, \ldots, p_{m}\right)$ and $v=\left(v_{3}, \ldots, v_{n}\right)$ is realizable on a closed orientable 2-manifold with Euler characteristic $\chi$ for some $p_{4}, v_{4} \in \mathbb{N}$ if and only if

$$
\begin{aligned}
\sum_{k=3}^{m}(4-k) p_{k}+\sum_{k=3}^{n}(4-k) v_{k}=4 \chi, & \\
\sum_{k=3}^{m} k p_{k} \equiv 0 & (\bmod 2), \\
p \neq[3,5] \quad \text { or } \quad v \neq\left[v_{4} \times 4\right] & \text { if } \chi=0, \\
p \neq\left[p_{4} \times 4\right] \quad \text { or } \quad v \neq[3,5] & \text { if } \chi=0 .
\end{aligned}
$$

Note that special cases arise in both theorems if the surface is a torus, i.e. if $\chi=0$. Izmestiev et al. [5] gave a simple argument using holonomy groups to explain why these special cases occur.

The rest of this section is devoted to find an easy characterization for when we cannot hope Question 1.3 to have a positive answer. Let $M$ be a polyhedral map on a surface $S$ with vertices $V$, edges $E$, and faces $F, p$-vector $p=\left(p_{3}, \ldots, p_{n}\right)$ and $v$-vector $v=\left(v_{3}, \ldots, v_{m}\right)$. Let $\chi=\chi(S)$ be the Euler characteristic of $S$ and $e:=|E|$. The two basic combinatorial results here are double-counting

$$
2 e=\sum_{k=3}^{m} k \cdot p_{k}=\sum_{k=3}^{n} k \cdot v_{k},
$$

and the Euler-Poincaré relation

$$
|V|-|E|+|F|=\chi .
$$

One can easily deduce from these relations, that the following two equivalent conditions are necessary for two sequences $p, v$ being the $p$ - and $v$-vector of a polyhedral map:

Proposition 2.6. Let $p, v$ be the $p$ - and $v$-vector of a polyhedral map on a surface $S$. Then (3) is true for some $e \in \mathbb{N}$ and the following equivalent conditions hold:

$$
\begin{aligned}
\sum_{k=3}^{m}(6-k) p_{k}+2 \sum_{k=4}^{n}(3-k) v_{k} & =6 \chi(S), \text { and } \\
\sum_{k=3}^{m}(4-k) p_{k}+\sum_{k=3}^{n}(4-k) v_{k} & =4 \chi(S)
\end{aligned}
$$


Equivalent here means, that together with $\sum_{k \geq 3} p_{k}=\sum_{k \geq 3} v_{k}$ each equation can be deduced from the other. If $p$ and $v$ satisfy these equations, we will call the pair $(p, v)$ admissible (on $S)$. We remark that we gain precisely the conditions of Theorems 2.4 and 2.5 .

In light of Question 1.3 and using the same arguments, it is not difficult to see, that we can always assume $p$ and $v$ to be admissible. Also important to note is, that from the same arguments we can derive similar conditions on $q$ and $w$ which have to be fulfilled in order for Question 1.3 to be answered in the positive. We will not go into the details here and simply state the cases resulting from these restrictions.

We will restrict our setting to $q=\left[q_{s} \times s, q_{l} \times l\right]$ having only two non-negative entries and $w=\left[w_{r} \times r\right]$ having one. Let us further assume that $\operatorname{gcd}\left(q_{s}, q_{l}\right)=1$. These conditions are quite natural, as any obstruction on finding a Eberhard-type theorem for some $q$ will also give an obstruction for $c \cdot q, c \in \mathbb{N}$. As stated above, not all values $s, l$, and $r$ can be obtained in this setting, only the following cases can occur:

$$
\begin{aligned}
& (s, r)=(3,3): \quad q=\left[q_{3} \times 3, q_{l} \times l\right], \quad w=[3], \quad q_{3}=\frac{l-6}{\operatorname{gcd}(l, 3)}, \quad q_{l}=\frac{3}{\operatorname{gcd}(l, 3)} \\
& (s, r)=(4,3): \quad q=\left[q_{4} \times 4, q_{l} \times l\right], \quad w=[3], \quad q_{4}=\frac{l-6}{\operatorname{gcd}(l, 2)}, \quad q_{l}=\frac{2}{\operatorname{gcd}(l, 2)} \\
& (s, r)=(5,3): \quad q=\left[q_{5} \times 5, q_{l} \times l\right], \quad w=[3], \quad q_{5}=l-6, \quad q_{l}=1 \\
& (s, r)=(3,4): \quad q=\left[q_{3} \times 3, q_{l} \times l\right], \quad w=[4], \quad q_{3}=l-4, \quad q_{l}=1 \\
& (s, r)=(3,5): \quad q=\left[q_{3} \times 3, q_{l} \times l\right], \quad w=[5], \quad q_{3}=3 l-10, \quad q_{l}=1
\end{aligned}
$$

We will answer the fourth case in this article. The full result by the author is the following:

Theorem 2.7 (Manecke [8], 2016). Let $q=\left[q_{s} \times s, q_{l} \times l\right], w=[r]$ as before. Then there exist inifitely many $c, d \in \mathbb{N}$ and a polyhedral map $M$ on a surface $S$ for all admissible sequences $(p, v)$ if and only if $\operatorname{gcd}(s, l)=1$ and if $s=r=3$, then $l<11$.

We close the section by noting that by duality this result gives also a full classification for $w$ having two non-zero entries and $q$ having only one.

\section{Construction}

Let $r \in \mathbb{N}$ be the valence of those vertices we are free to add to a polyhedral map. All constructions later in this article will utilize the concept of replacing each face of a polyhedral map with a larger patch. It can be quite challenging to see whether the resulting structures fit together. This section introduces the necessary formalism for these kinds of constructions. All statements are presented without proof, all proofs can be found in 8]. Note that throughout this section we allow 2 -valent vertices in special maps we call patches.

Definition 3.1 (Patch). A map $\mathcal{P}$ on the Euclidean plane with possibly 2-valent vertices on the unbounded outer face is called a patch. The vertices and edges of the outer face form the boundary $\partial \mathcal{P}$ of the patch. A patch is an r-patch, if each of its vertices except the ones on the boundary is $r$-valent, while for the valence $\operatorname{deg}(v)$ of a vertex $v$ on the outer face $2 \leq \operatorname{deg}(v) \leq r$ holds. The p-vector of $\boldsymbol{a}$ patch is the sequence $\left(p_{3}, p_{4}, \ldots\right)$, where $p_{k}$ denotes the number of $k$-gonal inner faces of the patch. 
We say that two $r$-patches $\mathcal{P}_{1}$ and $\mathcal{P}_{2}$ fit together along a path $v_{1}-\cdots-v_{n}$ on $\partial \mathcal{P}_{1}$ and $u_{1}-\cdots-u_{n}$ on $\partial \mathcal{P}_{2}$, if, after gluing them together such that $v_{i}=u_{n+1-i}$ the resulting patch is still an $r$-patch. Define $w(v):=\operatorname{deg}(v)-1$. Then the condition for fitting together is just $w\left(v_{i}\right)+w\left(u_{n+1-i}\right)=r$ for all $i \in\{2, \ldots, n-1\}$ and $w\left(v_{i}\right)+w\left(u_{n+1-i}\right) \leq r, w\left(v_{n+1-i}\right)+w\left(u_{i}\right) \leq r$. We say a tuple $\left(w_{1}, \ldots, w_{n}\right)$ is self-fitting, if $w_{i}+w_{n+1-i}=r$ for all $i \in\{1, \ldots, n\}$.

Essential for our constructions will be $w$-expansions. We will use them, when we replace all $k$-gons in a polyhedral map with larger structures.

Definition 3.2. Let $w=\left(w_{1}, \ldots, w_{n}\right) \in \mathbb{N}^{n}$. A w-expansion of an r-patch $\mathcal{P}$ with boundary $\partial \mathcal{P}=v_{1}-v_{2}-\cdots-\cdots-v_{m}-v_{1}$ is an $r$-patch $\mathcal{P}^{\prime}$ with boundary

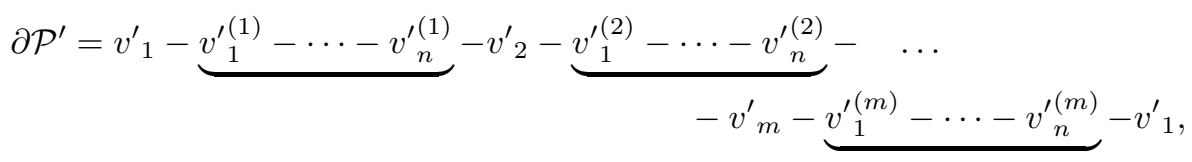

such that $w\left(v_{i}\right)=w\left(v_{i}^{\prime}\right)$ and $w\left({v^{\prime}}_{j}^{(i)}\right)=w_{j}$ for all $i \in\{1, \ldots, m\}, j \in\{1, \ldots, n\}$. We call the vertices $v_{i}^{\prime}$ corner vertices and the vertices $v_{j}^{\prime(i)}$ side vertices. Furthermore, a patch is called $w$-k-gonal, if it is the $w$-expansion of the patch consisting of only a $k$-gon, i.e. if $w\left(v_{i}^{\prime}\right)=1$ for $i \in\{1, \ldots, k\}$.

Using this notation we describe the following construction scheme:

Algorithm 3.3. Input: A map on a surface $S$ with p-vector $p$, v-vector $v$, underlying graph $G=(V, E)$ and faces $F$.

A self-fitting tuple $w=\left(w_{1}, \ldots, w_{n}\right)$.

For each $k$-gonal face $f \in F$ a w-k-gonal r-patch $\mathcal{P}(f)$ with p-vector $p^{(f)}$.

Output: A map on $S$ with v-vector $v+d \cdot[r]$ for some $d \in \mathbb{N}$ and $p$-vector $\sum_{f \in F} p^{(f)}$. Description: Divide each edge $e \in E$ in the embedding of $G$ in $S$ by $n$ vertices and draw into each face $f$ the dedicated $r$-patch $\mathcal{P}(f)$ such that the corner vertices of $\mathcal{P}(f)$ coincide with the original vertices $V$ and the side vertices are the new vertices added by the subdivision, see Fig. 1. Here we use the fact, that our surfaces are oriented and assume that all patches are glued with the same orientation. These patches form a combined graph, which is embedded by construction into $S$ (there is a homeomorphism between each subdivided face $f \in F$ and the corresponding patch $\mathcal{P}(f))$. It is straightforward to see, that this gives a map with the desired properties.

As previously stated, these definitions are used to formalize the construction step "replace each face with a patch". Up until now, there is no requirement explicitly stated on the interior of the patch. If we expect the result of such a construction to be a polyhedral map, further conditions have to be met. Additionally, when using Algorithm 3.3 we have the problem of assigning a patch for each face of the map. While we might need only one type of patch for a $k$-gon for each $k \geq 3$, we could still have to deal with a huge amount of values of $k$. We now want to define a construction scheme for patches for arbitrary $k$ which additionally allow to create polyhedral maps, even from non-polyhedral ones.

Definition 3.4. Let $\mathcal{P}$ be an r-patch with boundary

$$
\begin{aligned}
\partial \mathcal{P}=i_{0}-i_{1}-\cdots-i_{m-1}-\left(i_{m}=o_{0}\right)-\cdots-o_{s}- & \cdots-o_{n-1}-\left(o_{n}=i_{m}^{\prime}\right) \\
& -i_{m-1}^{\prime}-\cdots-i_{1}^{\prime}-i_{0}^{\prime}-i_{0}
\end{aligned}
$$



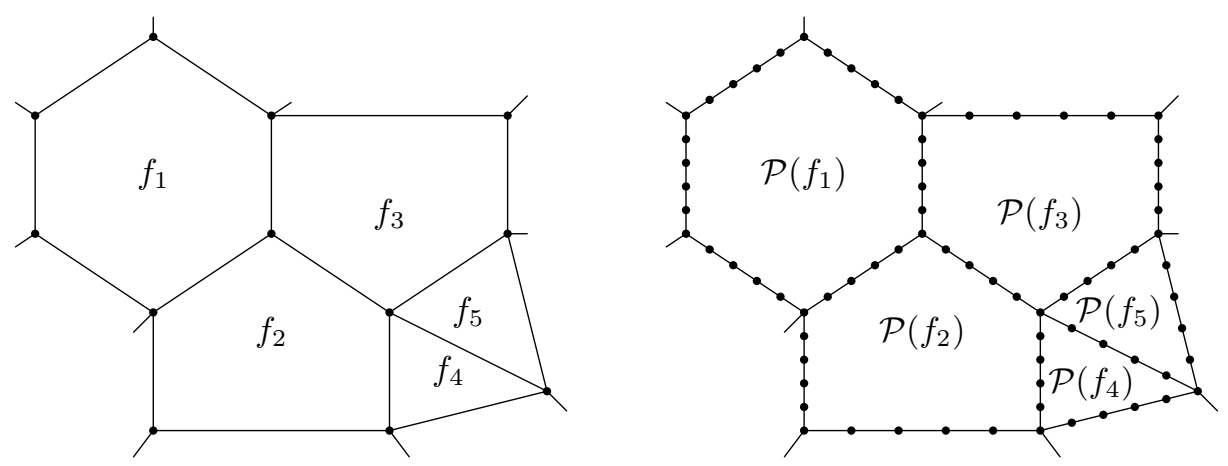

Figure 1.

$\left(i_{m}\right.$ and $o_{0}$ denote the same vertex, the same holds for $o_{n}$ and $i_{m}^{\prime}$ ), $1 \leq s<n$, $m>0$, such that:

- $w\left(i_{0}\right)+w\left(i_{0}^{\prime}\right)=r-1$,

- $\mathcal{P}$ fits to itself along $i_{1}-\cdots-i_{m-1}$ and $i_{m-1}^{\prime}-\cdots-i_{1}^{\prime}$,

- $w\left(o_{s}\right)=1$, and

- $\left(w\left(o_{s+1}\right), \ldots, w\left(o_{n-1}\right), w\left(o_{n}\right)+w\left(o_{0}\right), w\left(o_{1}\right), \ldots, w\left(o_{s-1}\right)\right)$ is a self-fitting tuple.

Such a patch will be called expansion patch with outer tuple $\left(w\left(o_{s+1}\right), \ldots\right.$, $\left.w\left(o_{n-1}\right), w\left(o_{n}\right)+w\left(o_{0}\right), w\left(o_{1}\right), \ldots, w\left(o_{s-1}\right)\right)$.

Example 3.5. We want to review the last definition with two examples. A hexagon can be interpreted as an expansion 3-patch $\mathcal{H}$ with outer tuple $\left(w_{\mathcal{H}}\left(o_{3}\right)+w_{\mathcal{H}}\left(o_{0}\right)\right.$, $\left.w_{\mathcal{H}}\left(o_{1}\right)\right)=(2,1)$, with vertices labeled according to Definition 3.4 in Fig. Q. Similarly two quadrangles which share a common edge build an expansion 4-patch $\mathcal{Q}_{2}$ with outer tuple $\left(w_{\mathcal{Q}_{2}}\left(o_{3}\right)+w_{\mathcal{Q}_{2}}\left(o_{0}\right), w_{\mathcal{Q}_{2}}\left(o_{1}\right)\right)=(2,2)$, as seen in the same figure.
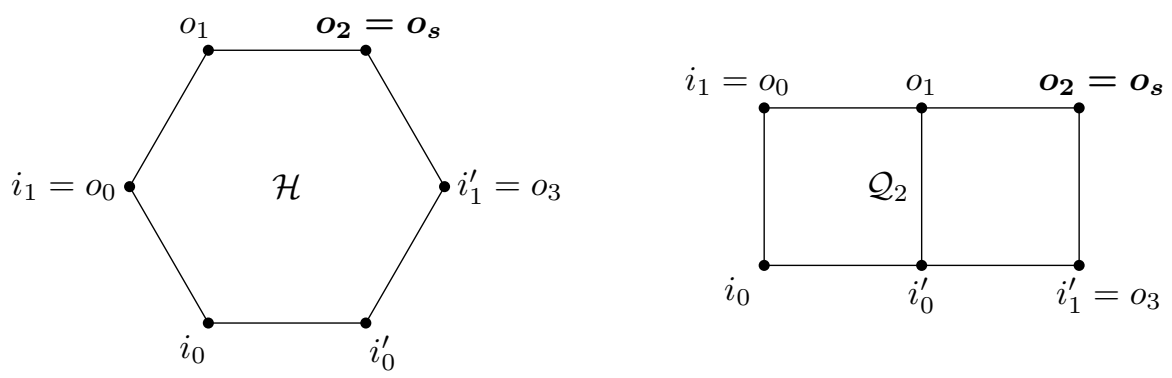

Figure 2. Two expansion patches

We want to pull apart this definition a bit to give a geometric intuition. The first thing to note is that by definition we are able to glue two copies of an expansion patch along the paths $i_{0}-\cdots-i_{m}$ and $i_{m}^{\prime}-\cdots-i_{0}^{\prime}$. When doing this the new patch has a boundary path $o_{s+1}-\cdots-o_{n-1}-\left(o_{n}=o_{0}\right)-o_{1}-\cdots-o_{s-1}$, which we require to be self-fitting. Therefore we can glue two of those patches along this boundary to get an even larger patch (see Fig. 3). 


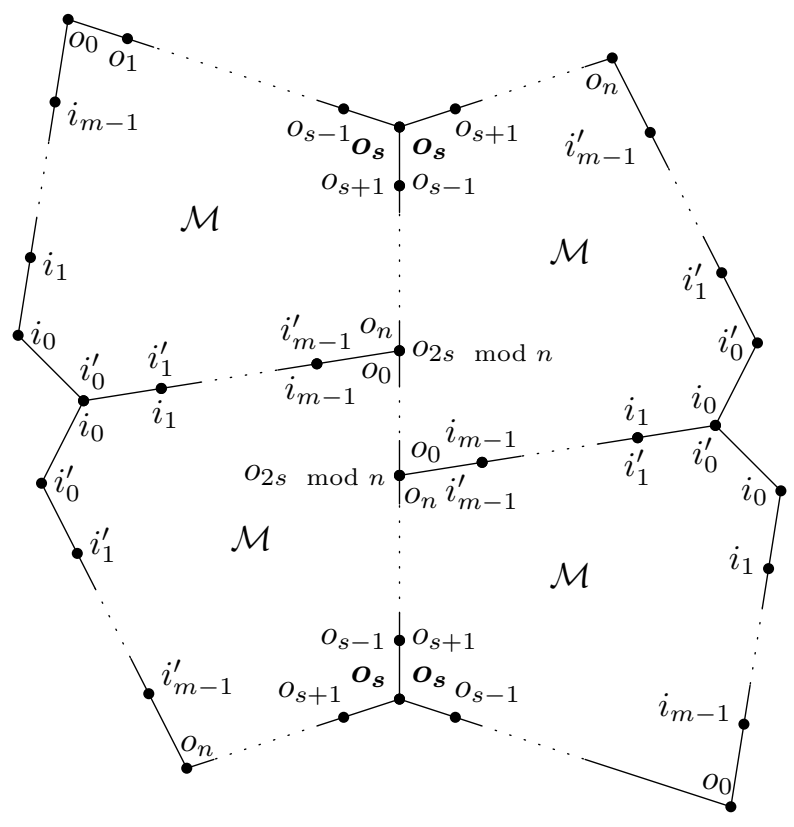

Figure 3. An edge patch

For an expansion patch $\mathcal{M}$, we want to call the patch obtained by gluing four copies of $\mathcal{M}$ as stated the edge patch of $\mathcal{M}$. An expansion patch will be said to have the polyhedral property if every two inner faces in the corresponding edge patch meet properly.

Example 3.6. The examples in Example 3.5 do in fact have the polyhedral property,

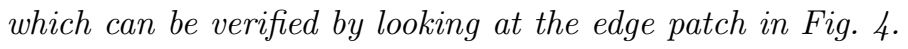
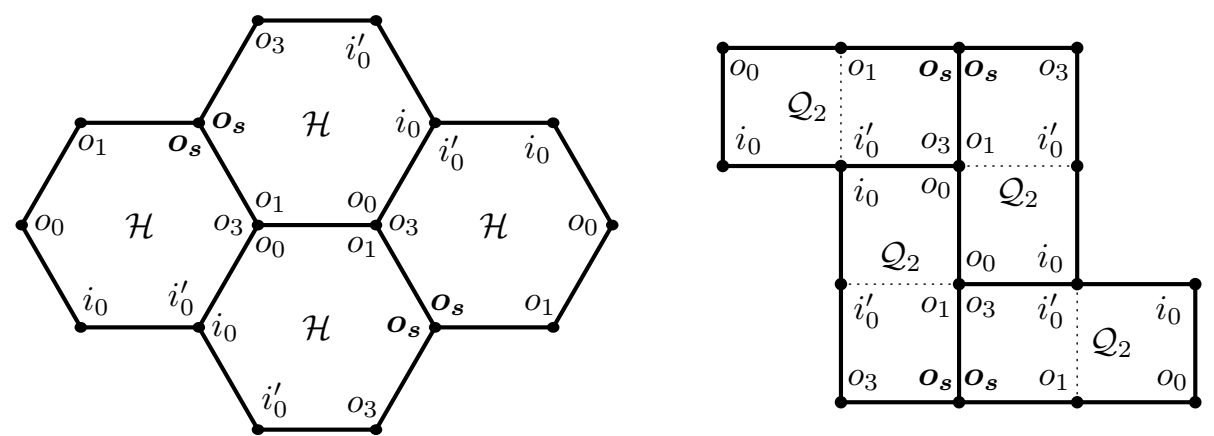

FIgURE 4. Two edge patches

Expansion patches will be our basic building block for all our constructive proofs. We can use them to obtain larger $o$ - $k$-gonal patches for any $k \geq 3$ :

Algorithm 3.7. Input: An expansion r-patch $\mathcal{M}$ with outer tuple o and p-vector $p$. 
Output: For every $k \geq 3$ an o-k-gonal r-patch $\mathcal{M}(k)$ with $p$-vector $[k]+k \cdot p$.

If $\mathcal{M}$ has the polyhedral property, then all inner faces of $\mathcal{M}(k)$ meet properly. Description: We construct $\mathcal{M}(k)$ from $k$ copies of $\mathcal{M}$ and a single $k$-gon. Let

$$
\begin{array}{r}
\partial \mathcal{P}=i_{0}-i_{1}-\cdots-\left(i_{m}=o_{0}\right)-o_{1}-\cdots-o_{s}-\cdots-o_{n-1}-\left(o_{n}=i_{m}^{\prime}\right)-\ldots \\
-i_{1}^{\prime}-i_{0}^{\prime}-i_{0}
\end{array}
$$

be the boundary of $\mathcal{M}$ as in Definition 3.4. We now form a larger patch by gluing the edge $\left\{i_{0}, i_{0}^{\prime}\right\}$ of each of the $k$ copies of $\mathcal{M}$ to an edge of the $k$-gon and also gluing the vertex associated to $i_{l}, 1 \leq l \leq m$ from one copy to the vertex associated to $i_{l}^{\prime}$ from the adjacent copy. Graphically speaking, we form a ring of $k$ patches of the form $\mathcal{M}$ around the $k$-gon. The p-vector of $\mathcal{M}(k)$ is therefore $[k]+k \cdot p$. We leave out the proof that the inner faces of $\mathcal{M}(k)$ meet properly in the case of $\mathcal{M}$ being polyhedral.

With these constructions at hand we can now finally design a scheme to create a polyhedral map from a non-polyhedral one.

Proposition 3.8. Given a map $M$ on an orientable closed 2-manifold $S$ and an expansion r-patch $\mathcal{M}$ with outer tuple o, Algorithm 3.3 returns a polyhedral map on $S$ when we take $\mathcal{P}(f)=\mathcal{M}(k)$ for each $k$-gonal face $f$ of $M$.
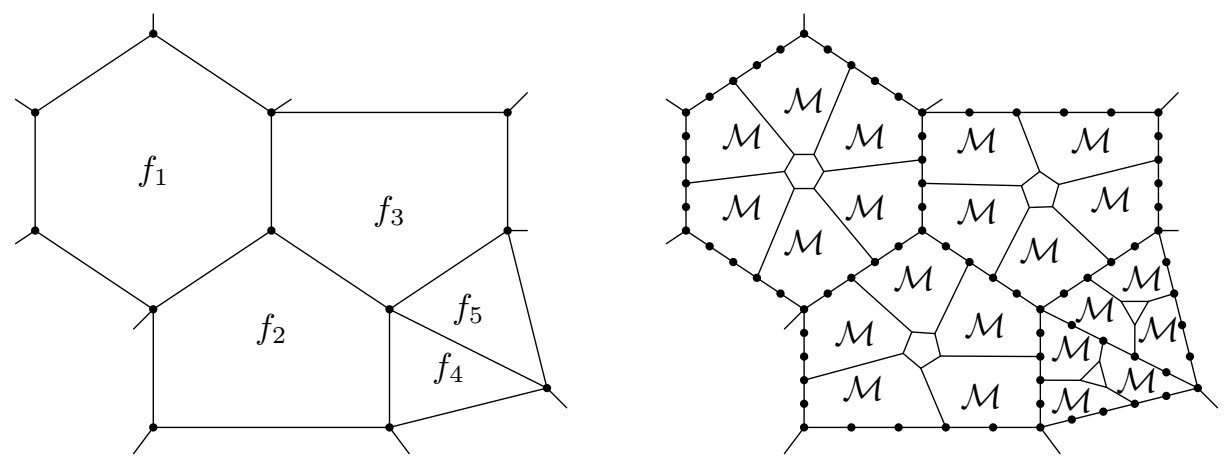

FiguRE 5. Algorithm 3.3 for $\mathcal{P}(f)=\mathcal{M}(k)$

Example 3.9. Using the expansion patches $\mathcal{H}$ and $\mathcal{Q}_{2}$ from Examples 3.5 and 3.6, we can construct from a polyhedral map a new one with arbitrarily many hexagons (or quadrangles) added, while inserting only 3-valent (or 4-valent) vertices. Given a map $M$ on a closed oriented 2-manifold, we can simply use Theorem 3.8 repeatedly on $M$ with either $\mathcal{H}$ or $\mathcal{Q}_{2}$ to get the desired result. The theorem inserts at least a single hexagon or quadrangle during each step (which is quite an understatement, the number of polygons added is by far larger), so repeating this step eventually leads to a map that has more than a specified amount of hexagons or quadrangles.

Putting all these constructions together, we can formulate a proof strategy for Question [1.3 in the case of $q=\left[q_{s} \times s, q_{l} \times l\right], w=[r]$. We state it for $r=4$ only, but the ideas carry over to $r=3$, too. 
Proposition 3.10. Let $r=4, p=\left(p_{3}, \ldots, p_{m}\right)$ and $v=\left(v_{3}, \ldots v_{n}\right)$ be an admissible pair of sequences. Let $w=[4]$ and $q=\left[q_{s} \times s, q_{l} \times l\right]$, where $s=3, l>4$, $q_{s}=l-4$ and $q_{l}=1$. Assume there exist

- an expansion r-patch $\mathcal{P}_{N}$ with outer tuple o consisting of s-gons and l-gons,

- an o-4-gonal r-patch $\mathcal{P}_{F}$ consisting of s-gons and l-gons, and

- an expansion $r$-patch $\mathcal{P}_{P}$ with the polyhedral property consisting of s-gons and l-gons.

Then there exists a polyhedral map on $S$ with $p$-vector $p+c \cdot q$ and $v$-vector $v+d \cdot w$ for infinitely many $c, d \in \mathbb{N}$.

Idea of the proof. Use Theorem 2.4 or Theorem 2.5 as a starting map and apply Proposition 3.10 for the given patches.

Remark 3.11. We will use Theorem 3.10 heavily in the next section. Therefore, we want to stress what is needed to check to see if the prerequisites of Theorem 3.10 are fulfilled. As stated, we need three $r$-patches, $\mathcal{P}_{N}, \mathcal{P}_{F}$ and $\mathcal{P}_{P}$, which are called in this manner for the rest of the article. The list of properties is:

- $\mathcal{P}_{N}, \mathcal{P}_{F}$ and $\mathcal{P}_{P}$ consist of only s-gons and l-gons and all inner vertices have valence $r$.

- $\mathcal{P}_{N}$ and $\mathcal{P}_{P}$ are expansion patches:

- $i_{0}$ and $i_{0}^{\prime}$ are in sum incident to $r-1$ faces,

- $i_{k}$ and $i_{k}^{\prime}$, are in sum incident to $r$ faces, $1 \leq k<m$,

- starting at the vertex $o_{s}$ and going in both directions for each pair of vertices $o_{s+k} \bmod n$ and $o_{s-k} \bmod n$ the identity $w_{\mathcal{P}_{X}}\left(o_{s+k} \bmod n\right)+$ $w_{\mathcal{P}_{X}}\left(o_{s-k} \bmod n\right)=r$ holds, where we "identify" $o_{n}$ with $o_{0}$. For ease of comparison we also state the outer tuple o for $\mathcal{P}_{N}$.

- $\mathcal{P}_{F}$ is o-4-gonal, i.e. if starting at some vertex and looking at the number of inner faces incident to this vertex we see the pattern

$$
1, o_{1}, \ldots o_{n}, 1, o_{1}, \ldots o_{n}, 1, o_{1}, \ldots o_{n}, 1, o_{1}, \ldots o_{n},
$$

where o is the outer tuple of $\mathcal{P}_{N}$.

- $\mathcal{P}_{P}$ has the polyhedral property. For this we provide the corresponding edge patch to make the verification easier.

\section{4-VALENT EBERHARD-TYPE THEOREMS With TRIANGLES}

In this section we want to prove 4 -valent Eberhard-type theorems with triangles, i.e. for $q=\left[q_{3} \times 3, q_{l} \times l\right], w=\left[w_{4} \times 4\right], l>4, \operatorname{gcd}\left(q_{3}, q_{l}\right)=1$.

For all the proofs, we want to use Theorem 3.10, therefore we need to have a construction scheme for patches with arbitrarily large $l$-gons. These we get by the next three constructions:

Algorithm 4.1. When we want to use this construction in this section we label an edge (the specified edge) with a square and point with arrows to a $k_{1}$-gon and a $k_{2}$-gon.

Input: A 4-patch with p-vector $p$ and a specified edge with exactly one vertex incident to some $k_{1}$-gon and the other vertex incident to some $k_{2}$-gon. We require that in the cyclic order around both end points, starting at the specified edge, the $k_{1}$-gon and the $k_{2}$-gon have the same position.

Output: A new 4-patch with p-vector $p-\left[k_{1}, k_{2}\right]+[2 k \times 3]+\left[k_{1}+k, k_{2}+k\right]$ for all $k \in \mathbb{N}$. 
If every two faces of the 4-patch meet properly, then this is carried over to the new patch.

Description: Using the replacement of the single edge as seen in Fig. [6 results in a new 4-patch with p-vector $p-\left[k_{1}, k_{2}\right]+[2 \times 3]+\left[k_{1}+1, k_{2}+1\right]$. The line on the left labeled with a square is the specified edge and the line on the right labeled with a square is a new edge that we can use to repeat the construction. Every time we use this construction we add two new triangles while increasing the number of vertices of the left and right polygon by one; doing this $k$ times gives the desired 4-patch. That all faces meet properly follows by induction as this property is preserved in each step.
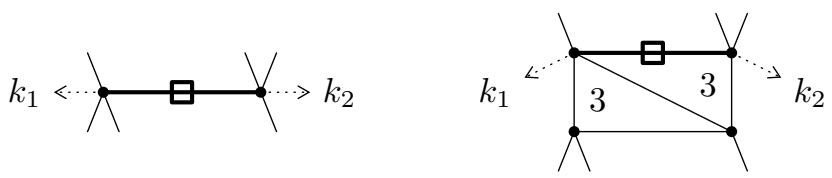

FiguRE 6.

Algorithm 4.2. When we want to use this construction in this section we label an edge (the specified edge) with a diamond and point with arrows to a $k_{1}$-gon and a $k_{2}$-gon.

Input: A 4-patch with p-vector $p$ and a specified edge which is the common edge of a $k_{1}$-gon and a $k_{2}$-gon.

Output: $A$ 4-patch with p-vector $p-\left[k_{1}, k_{2}\right]+[(6 k) \times 3]+\left[k_{1}+3 k, k_{2}+3 k\right]$ for all $k \in \mathbb{N}$.

Description: Using the replacement of the single edge as seen in Fig. 7 results in a new 4-patch with p-vector $p-\left[k_{1}, k_{2}\right]+[(6 k) \times 3]+\left[k_{1}+3, k_{2}+3\right]$. The line on the left labeled with a diamond is the specified edge and the line on the right labeled with a diamond is a new edge which we can use to repeat the construction. Every time we use this construction we add six triangles while increasing the number of vertices of the left and right polygon by three; doing this $k$ times gives the desired 4-patch.
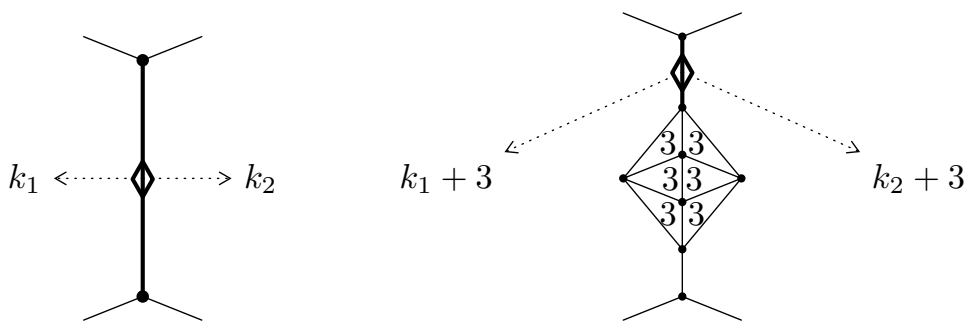

FIGURE 7.

Algorithm 4.3. When we want to use this construction in this section we encircle a vertex (the specified vertex) and point with arrows to a $k_{1}$-gon and a $k_{2}$-gon.

Input: A 4-patch with p-vector $p$ and a specified vertex which is adjacent to both $k_{1}$-gon and a $k_{2}$-gon which do not share an edge containing this vertex. 
Output: A new 4-patch with p-vector $p-\left[k_{1}, k_{2}\right]+[(6 k) \times 3]+\left[k_{1}+3 k, k_{2}+3 k\right]$ for all $k \in \mathbb{N}$.

Description: Using the replacement of the vertex as seen in Figure 8 results in a new 4-patch with p-vector $p-\left[k_{1}, k_{2}\right]+[(6 k) \times 3]+\left[k_{1}+3, k_{2}+3\right]$. The encircled vertex on the left is the specified vertex and the encircled vertex on the right is a new vertex which we can use to repeat the construction. Every time we use this construction we add six triangles while increasing the number of vertices of the left and right polygon by three; doing this $k$ times gives the desired 4-patch.

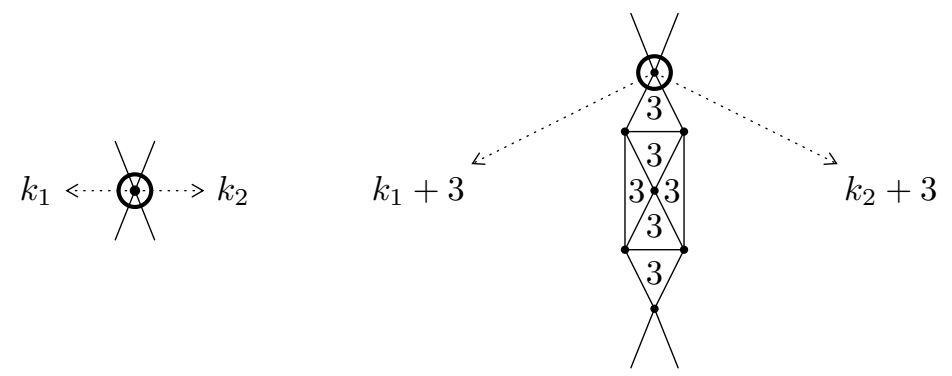

FiguRE 8.

With our whole machinery at work, we can now state the proofs of our main theorems easily:

Proof of Theorem 1.4. An expansion 4-patch $\mathcal{P}_{N}$ with outer tuple $o=(1,2,1,3$, 2,3 ) is shown in Fig. 9(a) and a corresponding o-4-gonal 4-patch $\mathcal{P}_{F}$ is shown in Fig. 9(d), both consisting of triangles and pentagons. By using Algorithm 4.1. Algorithm 4.2 and Algorithm 4.3 as indicated we get 4-patches consisting of only triangles and $(3 k+5)$-gons, $k \in \mathbb{N}$. We can see in Fig. 9(b) that $\mathcal{P}_{N}$ has the polyhedral property, thus we can apply Theorem 3.10 with $\mathcal{P}_{P}:=\mathcal{P}_{N}$.

Proof of Theorem 1.5. An expansion 4-patch $\mathcal{P}_{N}$ with outer tuple $o=(2,2,3,2,1$, 3, 2, 1,2,2) is shown in Fig. 9(a) and a corresponding $o$-4-gonal 4-patch $\mathcal{P}_{F}$ is shown in Fig. 9(c), both of which consist of only triangles and heptagons. By using Algorithm 4.1. Algorithm 4.2 and Algorithm 4.3 as indicated we get 4-patches consisting of only triangles and $(3 k+7)$-gons, $k \in \mathbb{N}$. We can reuse the 4-patch $\mathcal{P}_{P}$ with the polyhedral property in Fig. 9(a) from the last theorem and after application of Algorithm 4.1 it likewise contains triangles and $(3 k+7)$-gons. Thus we can apply Theorem 3.10 


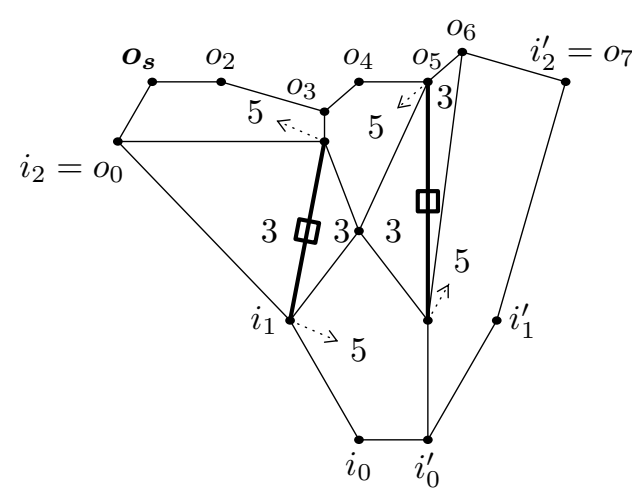

(a) $\mathcal{P}_{N}=\mathcal{P}_{P}$

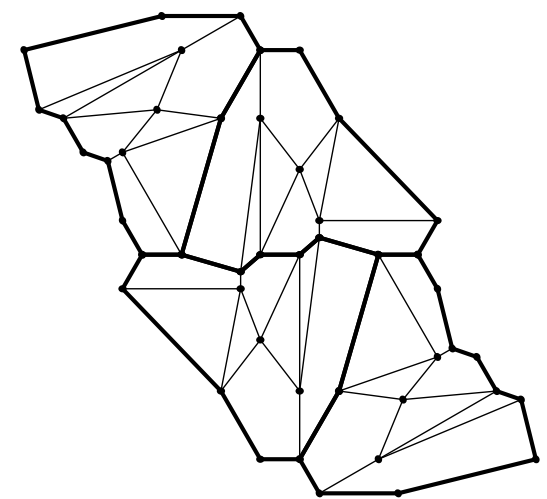

(b) Edge patch of $\mathcal{P}_{N}$

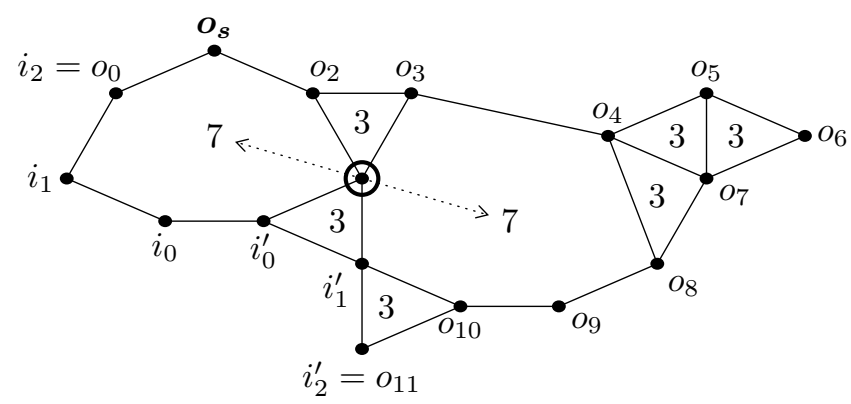

(c) $\mathcal{P}_{N}$

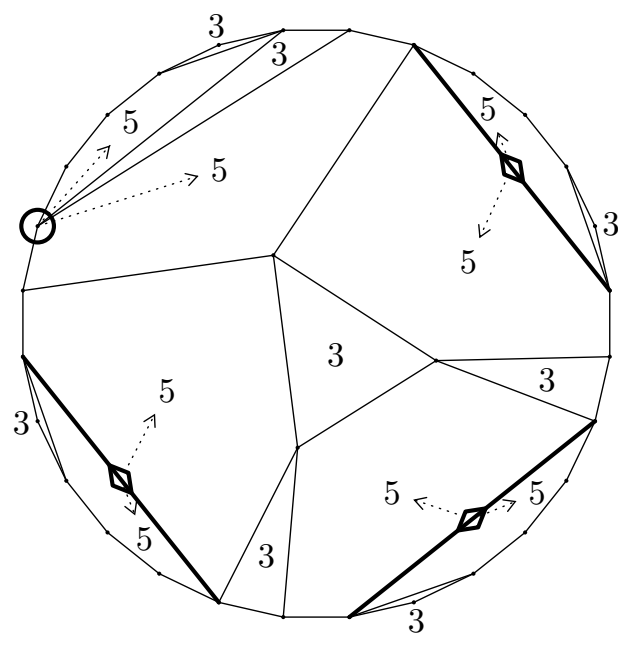

(d) $\mathcal{P}_{F}$

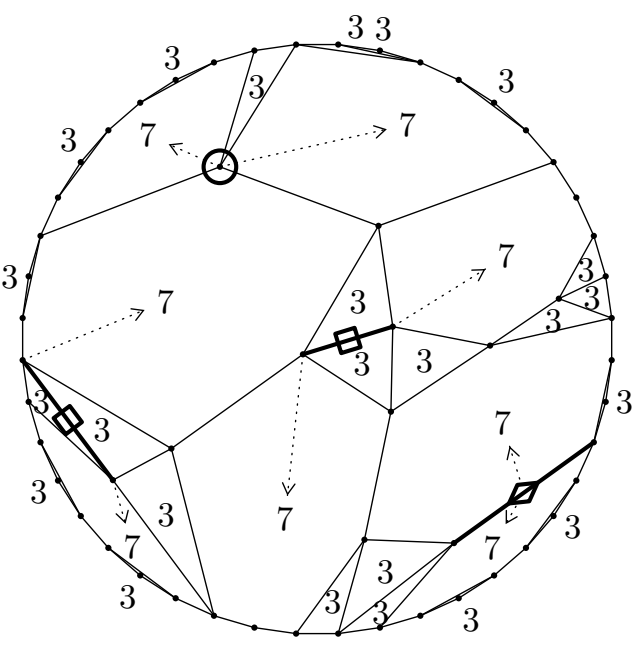

(e) $\mathcal{P}_{F}$

Figure $9 . \mathcal{P}_{N}=\mathcal{P}_{P}$, the edge patch of $\mathcal{P}_{N}$ and $\mathcal{P}_{F}$ 


\section{REFERENCES}

[1] D. W. Barnette, E. Jucovič and M. Trenkler, Toroidal Maps With Prescribed Types Of Vertices And Faces, Mathematika 20, 82 (1971).

[2] M. DeVos, A. Georgakopoulos, B. Mohar and R. Šámal, An Eberhard-like theorem for pentagons and heptagons, Discrete \& Computational Geometry 44, 931 (2010).

[3] B. Grünbaum, Planar maps with prescribed types of vertices and faces, Mathematika 16, 28 (1969).

[4] B. Grünbaum et al., Convex Polytopes (Springer, 2003).

[5] I. Izmestiev, R. B. Kusner, G. Rote, B. Springborn and J. M. Sullivan, There is no triangulation of the torus with vertex degrees $5,6, \ldots, 6,7$ and related results: Geometric proofs for combinatorial theorems, Geometriae Dedicata 166, 15 (2013).

[6] S. Jendrol' and E. Jucovič, Generalization of a theorem by V. Eberhard, Mathematica Slovaca 27, 383 (1977).

[7] E. Jucovič and M. Trenkler, A theorem on the structure of cell-decompositions of orientable 2-manifolds, Mathematika 20, 63 (1973).

[8] S. Manecke, New Eberhard-like theorems, Master's thesis, Technische Universität Dresden, (2016).

[9] J. Zaks, The analogue of Eberhards theorem for 4-valent graphs on the torus, Israel Journal of Mathematics 9, 299 (1971).

FB 12 - Institut für Mathematik, Goethe-Universität Frankfurt, Robert-Mayer-Str. 10, D-60325 Frankfurt am Main, Germany, E-mail: manecke@math.uni-Frankfurt.de 